\title{
Clinicopathological and prognostic value of long noncoding RNA SNHG7 in cancers: a meta-analysis and bioinformatics
}

\author{
June Wang ${ }^{1,}$, , Shenlin $\mathrm{Du}^{3,{ }^{*}}$, Chen Wang ${ }^{4,}{ }^{*}$, Zinian $\mathrm{Zhu}^{3}$, Baocheng $\mathrm{Xie}^{2}$, Bashan Zhang ${ }^{3, \&}$ \\ ${ }^{1}$ Central Laboratory, Affiliated Dongguan Hospital, Southern Medical University, Dongguan, China \\ ${ }^{2}$ Department of Pharmacy, Affiliated Dongguan Hospital, Southern Medical University, Dongguan, China \\ ${ }^{3}$ Clinical Laboratory, Affiliated Dongguan Hospital, Southern Medical University, Dongguan, China \\ ${ }^{4}$ Center for Gene Diagnosis, Zhongnan Hospital of Wuhan University, Wuhan, China \\ *Equal contribution
}

Correspondence to: Baocheng Xie, Bashan Zhang; email: baochengxie@126.com, https://orcid.org/0000-0002X-7109-402; zhangbs dgsrmyy@126.com

Keywords: IncRNA, SNHG7, cancer, clinical parameters, prognosis

Received: December 27, $2020 \quad$ Accepted: October 18, 2021

Published: October 29, 2021

Copyright: (C) 2021 Wang et al. This is an open access article distributed under the terms of the Creative Commons Attribution License (CC BY 3.0), which permits unrestricted use, distribution, and reproduction in any medium, provided the original author and source are credited.

\section{ABSTRACT}

The long intergenic non-coding RNA SNHG7 has been reported to be abnormally expressed in many types of cancer, the results remain controversial. In this study, a meta-analysis was performed to evaluate the clinicopathologic and prognostic value of SNHG7 in cancers. Electronic databases of PubMed, Web of Science, Cochrane Library and Embase were used to search relevant studies. A combined hazard ratio (HR) and its corresponding $95 \%$ confidence interval $(\mathrm{Cl})$ were used to assess the association between SNHG7 expression and prognosis in cancer patients. Pooled odds ratio (OR) and $95 \% \mathrm{Cl}$ were calculated to elaborate the association between SNHG7 expression and clinicopathological features in cancers. Besides, the data from The Cancer Genome Atlas (TCGA) dataset was used to validate the results. In total, eighteen studies compromising 1303 participants were enrolled in this analysis. The pooled results showed increased SNHG7 expression could predict unfavorable overall survival $(\mathrm{OS})(\mathrm{HR}=1.75,95 \% \mathrm{Cl}=1.52-2.02, \mathrm{P}=\mathbf{0 . 0 0 0})$. Analysis stratified by followup time, cancer types, analysis types, sample sizes and cut off further verified the prognostic value of SNHG7. Additionally, elevated SNHG7 expression was correlated with TNM stage (OR: $3.31,95 \% \mathrm{Cl}=2.29-4.80$, $P=0.000)$, lymph node metastasis $(O R=3.32,95 \% \mathrm{Cl}=1.61-6.83, P=0.004)$, and tumor differentiation $(O R=1.92,95 \% \mathrm{Cl}=1.22-3.03, P=0.005)$ in patients with cancers. Excavation of TCGA dataset valuated that SNHG7 was upregulated in some cancers and predicted worse OS, which partially confirmed our results in this meta-analysis.

\section{INTRODUCTION}

Cancer has been recognized as one of the most serious public health problems on a global scale. Due to changes in environmental factors and socio-economic development, the incidence and mortality of cancer are rising rapidly $[1,2]$. It's predicted that people affected by cancer will reach approximately 11.4 million by 2020, and one-third of the population will get cancer in their lifetime [3]. Despite enormous progress in the management and therapy of tumors over the past decade, the five-year survival rate for tumors is still not optimistic, mainly because many cancer patients have entered an advanced stage when they were diagnosed [4]. Therefore, it remains a high unmet need to discover new and effective biomarkers at an early stage to reduce cancer-related mortality.

Long non-coding RNAs (lncRNAs) which are a group of >200 nucleotides length RNA molecules do not contain functional open reading frame [5, 6]. Many lncRNAs defined as oncogenes and tumor suppressor 
genes in various types of cancer, effect on the cancer cell proliferation and apoptosis and involve in cancer invasion and metastasis [7-9]. For example, highly expressed lncHOTAIR could regulate cancer differentiation, distant metastasis in colorectal cancer tissues and closely related to TNM stage [10]. It has been reported that overexpressed LncSTCAT16 is a potent molecular target for inhibition of the cell proliferation and invasion in gastric cancer [11]. The up-regulation of LncPANDAR which promotes cell proliferation has been proved to related to poor prognosis of cervical cancer [12]. Besides, many researchers believed that some of IncRNAs could become potential therapeutic targets for monitoring cancer, predicting prognosis and evaluation of treatment effect $[13,14]$.

Small nucleolar RNA host gene 7 (SNHG7) has been regarded as a potential oncogene which is located on the long arm of chromosome 9 at band q34.3. It has been found that dysregulation of SNHG7 associated with growth and progress of different kinds of cancer, such as breast cancer, pancreatic cancer, hepatocellular carcinoma and cervical cancer [15-18]. Furthermore, a growing number of studies have explored the prognostic value of SNHG7 in various cancers, and suggested it can be exploited as a possible biomarker and therapeutic target for helping to improve diagnosis of cancer patients [19-23]. However, in current studies reporting the prognostic value of SNHG7 were subject to some qualifications, such as small sample size and contentious outcomes. Therefore, this quantitative meta-analysis cooperated with bioinformatics data was carried out to elucidate the prognostic value and clinicopathologic characteristics of SNHG7 in cancers.

\section{MATERIALS AND METHODS}

\section{Literature searching strategies}

For screening out all relevant studies up to August 2020, we conducted the online search of PubMed, Web of Science, Cochrane Library, and Embase. The keywords and $\mathrm{MeSH}$ terms used for the search were as follows: ("long non-coding RNA 7" or "SNHG7" or "small RNA host gene 7" or "IncSNHG7") and ("neoplasm" OR "tumor" or "carcinoma" or "cancer") and ("outcome" or "prognostic" or "prognosis"). Through searching other references cited by these retrieval studies, more related potential records were identified.

\section{Inclusion and exclusion criteria}

The inclusion criteria for eligible studies were as follows: (a) measuring the expression of SNHG7; (b) valuable data that could reflect the relationship between SNHG7 level and clinicopathological features or prognosis; (c) sufficient data for obtaining the values of HRs and CIs to judge the survival outcomes. The exclusion criteria were as follows: (a) repetitive research; (b) studies unrelated to cancer; (c) non-human studies; (d) unable to get the HRs and 95\%CI from raw data; (e) case reports, comment, and reviews.

\section{Quality evaluation and data extraction}

Two reviewers (JEW and CW) extracted the data independently from all identified records. The article general information, tumor type, sample type, the number of involved patients, detection method, followup time, cut off value, outcome measure, hazard rate and SNHG7 evaluation score were extracted from each study. Some articles directly provided at least one of three values of OS, DFS and PFS, while we had to use Engauge Digitizer version 4.1 to obtain the above data from the rest of articles according to the method described by Tierney [23]. All of the selected studies with the Newcastle-Ottawa Scale (NOS) score of 7 or above were considered highquality articles [24].

\section{Bioinformatics validation}

We exploited online analysis software the Gene Expression Profiling Interactive Analysis (GEPIA) to further explore the differential expression of SNHG7 among cancer tissues. All data are based on The Cancer Genome Atlas (TCGA) database. and We set P $<0.01$ as the cut-off value to compare TCGA various neoplasms data with normal group. In order to assess the correlation between SNHG7 expression and OS, we've chosen Kaplan-Meier method and log-rank test to calculate the survival analysis.

\section{Statistical analysis}

All statistical analyses were completed by the STATA software version 15.0. The chi-squared Q test and the $I^{2}$ statistic were used to observe the heterogeneity among studies. If the significant heterogeneity existed $\left(P<0.05\right.$ for Chi-squared test or $\left.I^{2}>50 \%\right)$, randomeffect model was used. If not, we used the fixed-effect model. Evaluation methods of the following correlation analyze, SNHG7 expression and prognosis in cancers: pooled HRs and 95\% CIs; SNHG7 expression and clinicopathological features: combined odds ratio (OR) and 95\% CIs; publication bias: funnel plots and Begg's test. By removing individual study in sequence, we used sensitivity analysis to verify that the results were credible and stable. Furthermore, we defined the $p$ value less than 0.05 as statistically significant. 


\section{RESULTS}

\section{Description of studies}

The detailed steps and processes of the article retrieval and selection were displayed in Figure 1. A total of 347 articles were revealed in the initial literature search. However, we selected 35 studies in strict accordance with inclusion and exclusion criteria. Subsequently, after screening the full texts of the remaining 35 articles, another 17 studies were excluded. Finally, only 18 articles included 1303 cancer patients were fully in conformity with the screening criteria and enrolled in the current meta-analysis [15-18, 22, 23, 25-36].

The selected studies were all conducted in China, and their main characteristics have been summarized in Table 1. Publication years ranged from 2018 to 2020 , and the sample sizes were between 30 and 162 . These eligible researches contained 1303 patients, involved 11 types of cancers. There were three hepatocellular carcinoma studies, three colorectal cancer studies, two prostate cancer studies, two neuroblastoma studies, two cervical cancer studies, one bladder cancer study, one gastric cancer study, one pancreatic cancer study, one osteosarcoma study, one hypopharyngeal cancer study, and one breast cancer study. In these studies, SNHG7 expression levels were detected in tumors using the qRT-PCR method. The enrolled patients were divided into two groups according to the expression of SNHG7: high expression and low expression groups. All studies examined the association of SNHG7 with OS, and one study reported PFS and DFS respectively. Five studies have directly given HR values, and other thirteen studies only provided the Kaplan-Meier survival curves for calculating the HR values indirectly. Moreover, the number of studies involved in distance metastasis (DM), lymph node metastasis (LNM), TNM stage, and tumor differentiation was $6,10,6$, and 6 respectively. All publications met the good quality in the NOS scoring system.

\section{The SNHG7 expression and disease prognosis}

A total of 18 eligible studies with a sample of 1303 patients were recruited to evaluate the expression level of SNHG7 on OS. The pooled HR was 1.75 (95\% CI: 1.52$2.02, P=0.000)$, which indicated that the high expression of SNHG7 was significantly related to shorter overall survival (Figure 2). Since there was no heterogeneity

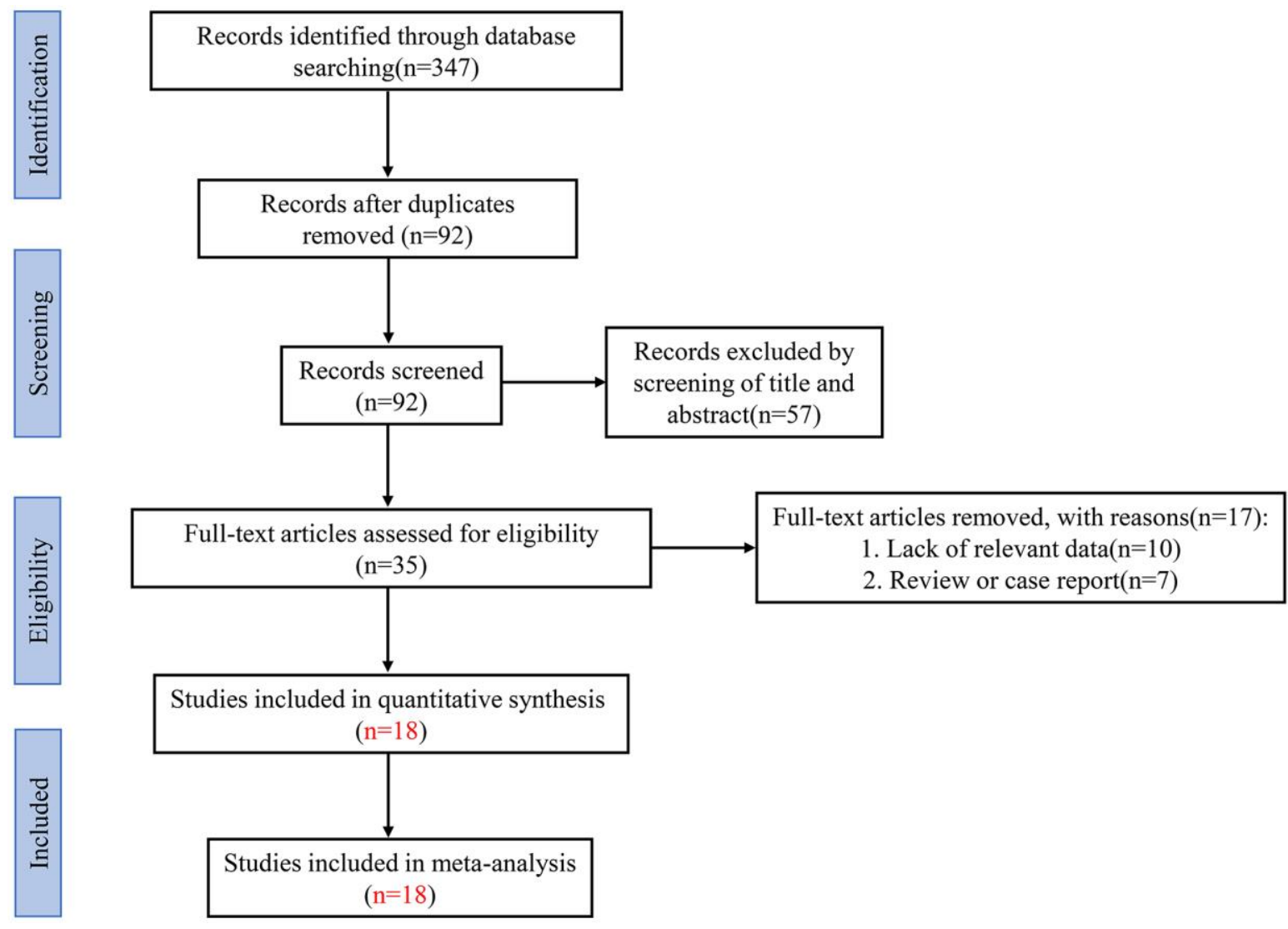

Figure 1. Flow diagram of the literature retrieval and selection in this meta-analysis. 
Table 1. Characteristics of studies in this meta-analysis.

\begin{tabular}{|c|c|c|c|c|c|c|c|c|c|c|c|}
\hline Study & Year & Country & Tumor types & $\begin{array}{l}\text { Sample } \\
\text { size }\end{array}$ & Sample & $\begin{array}{c}\text { Detection } \\
\text { method }\end{array}$ & $\begin{array}{c}\text { Cut off } \\
\text { value }\end{array}$ & $\begin{array}{c}\text { Follow-up } \\
\text { (year) }\end{array}$ & $\begin{array}{l}\text { Outcome } \\
\text { measures }\end{array}$ & $\begin{array}{l}\text { Extract } \\
\text { method }\end{array}$ & NOS \\
\hline Jia & 2020 & China & neuroblastoma & 45 & tissues & qRT-PCR & mean & 5 years & OS & K-M Curve & 8 \\
\hline Shen & 2020 & China & hepatocellular carcinoma & 100 & tissues & qRT-PCR & mean & 5 years & PFS OS & K-M Curve & 7 \\
\hline Zhang & 2020 & China & $\begin{array}{c}\text { synchronous colorectal liver } \\
\text { metastasis }\end{array}$ & 96 & serum & qRT-PCR & median & 3 years & OS & Reported & 8 \\
\hline $\mathrm{Wu}$ & 2020 & China & cervical cancer & 51 & tissues & qRT-PCR & median & 5 years & OS & K-M Curve & 7 \\
\hline $\mathrm{Xia}$ & 2020 & China & prostate cancer & 127 & tissues & qRT-PCR & mean & 5 years & OS & Reported & 8 \\
\hline Zhang & 2020 & China & gastric cancer & 162 & tissues & qRT-PCR & median & 8 years & OS & Reported & 9 \\
\hline Zeng & 2019 & China & cervical cancer & 60 & tissues & qRT-PCR & NA & 5 years & OS & Reported & 8 \\
\hline Yang & 2019 & China & hepatocellular carcinoma & 80 & tissues & qRT-PCR & median & 5 years & OS & K-M Curve & 8 \\
\hline Yao & 2019 & China & hepatic carcinoma & 40 & tissues & qRT-PCR & median & 3 years & OS & K-M Curve & 7 \\
\hline Cheng & 2019 & China & pancreatic cancer & 40 & tissues & qRT-PCR & NA & 3.5 year & OS & K-M Curve & 7 \\
\hline $\mathrm{Wu}$ & 2019 & China & hypopharyngeal cancer & 73 & tissues & qRT-PCR & median & 5 years & OS & K-M Curve & 8 \\
\hline Chi & 2019 & China & neuroblastoma & 92 & tissues & qRT-PCR & mean & 5 years & OS & K-M Curve & 8 \\
\hline Luo & 2018 & China & breast cancer & 72 & tissues & qRT-PCR & NA & 6.5 years & OS & K-M Curve & 7 \\
\hline Chen & 2019 & China & bladder cancer & 92 & tissues & qRT-PCR & NA & 5 years & OS & K-M Curve & 8 \\
\hline Deng & 2018 & China & osteosarcoma & 30 & tissues & qRT-PCR & median & 5 years & OS & K-M Curve & 7 \\
\hline $\mathrm{Li}$ & 2018 & China & colorectal cancer & 53 & tissues & qRT-PCR & NA & 5 years & OS DFS & Reported/ K-M Curve & 8 \\
\hline Qi & 2018 & China & prostate cancer & 42 & tissues & qRT-PCR & NA & 5 years & OS & K-M Curve & 7 \\
\hline Shan & 2018 & China & colorectal cancer & 48 & tissues & qRT-PCR & median & 5.5 years & OS & K-M Curve & 7 \\
\hline
\end{tabular}

between the studies $\left(I^{2}=0.0 \%, P=0.534\right)$, a fix-effect model was applied to analyze the HR and $95 \%$ CI of OS. Our analysis showed that the up-regulated SNHG7 expression had a positive correlation with the worse overall survival in cancer patients.

In order to further explore the correlation between SNHG7 expression and the OS, all included patients were classified, based on follow-up time, cancer types, analysis types, sample sizes and cut off, to perform the subgroup analysis of OS.

As showed in Table 2, a strong correlation was discovered between increased expression of SNHG7 and poor OS in digestive system cancers (HR $=1.64,95 \%$ $\mathrm{CI}=1.37-1.95, P<0.000)$, other cancers $(\mathrm{HR}=2.01$,

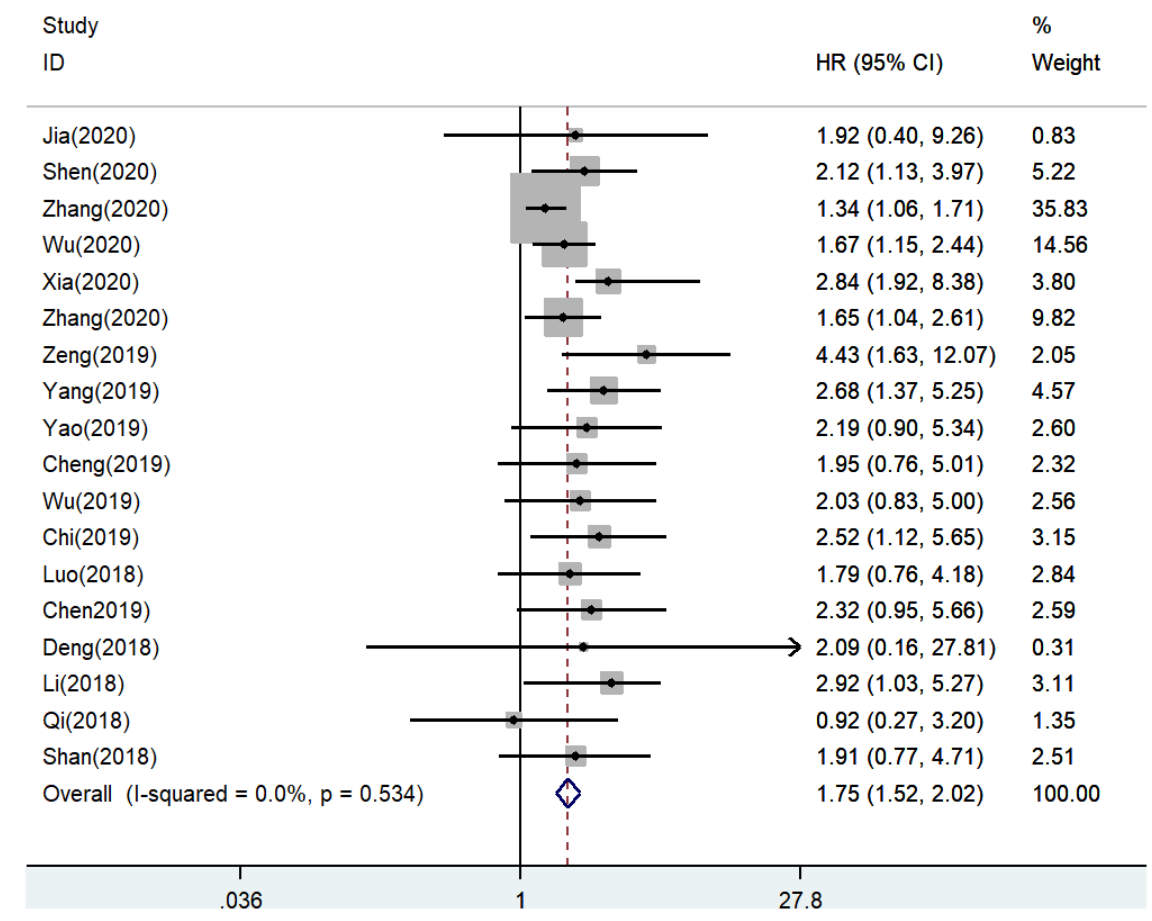

Figure 2. Forest plot for the association between SNHG7 expression with overall survival (OS). 
Table 2. Subgroup analysis of SNHG7 expression and overall survival (OS) in cancer patients.

\begin{tabular}{|c|c|c|c|c|c|c|c|}
\hline \multirow{2}{*}{ Subgroup analysis } & \multirow{2}{*}{ No. of studies } & \multirow{2}{*}{ No. of patients } & \multirow{2}{*}{$\begin{array}{c}\text { Pooled } \\
\text { HR(95\% CI })\end{array}$} & \multirow{2}{*}{$P$} & \multicolumn{2}{|c|}{ Heterogeneity } & \multirow{2}{*}{ Model } \\
\hline & & & & & $I^{2}(\%)$ & $P$-value & \\
\hline OS & 18 & 1303 & $1.75(1.52-2.02)$ & 0.000 & 0 & 0.534 & Fixed \\
\hline \multicolumn{8}{|l|}{ Follow-up time } \\
\hline$\geq 5$ years & 15 & 1127 & $2.03(1.69-2.45)$ & 0.000 & 0 & 0.865 & Fixed \\
\hline$<5$ years & 3 & 176 & $1.42(1.13-1.77)$ & 0.002 & 0 & 0.462 & Fixed \\
\hline \multicolumn{8}{|l|}{ Tumor type } \\
\hline Digestive system cancer & 8 & 619 & $1.64(1.37-1.95)$ & 0.000 & 11.7 & 0.339 & Fixed \\
\hline Others & 10 & 684 & $2.01(1.57-2.57)$ & 0.000 & 0 & 0.721 & Fixed \\
\hline \multicolumn{8}{|l|}{ HR estimation method } \\
\hline Multivariate & 5 & 498 & $2.06(1.37-3.10)$ & 0.001 & 61.8 & 0.033 & Random \\
\hline K-M Curve & 13 & 805 & $1.95(1.58-2.41)$ & 0.000 & 0 & 0.989 & Fixed \\
\hline \multicolumn{8}{|l|}{ Number of patients } \\
\hline$\geq 80$ & 7 & 749 & $1.66(1.39-1.99)$ & 0.000 & 34.2 & 0.167 & Fixed \\
\hline$<80$ & 11 & 554 & $1.94(1.52-2.47)$ & 0.000 & 0 & 0.838 & Fixed \\
\hline \multicolumn{8}{|l|}{ Cut-off } \\
\hline Mean & 4 & 364 & $2.39(1.61-3.56)$ & 0.000 & 0 & 0.932 & Fixed \\
\hline Median & 8 & 580 & $1.58(1.33-1.87)$ & 0.000 & 0 & 0.626 & Fixed \\
\hline NA & 6 & 359 & $2.27(1.55-3.31)$ & 0.000 & 0 & 0.475 & Fixed \\
\hline
\end{tabular}

95\% CI $=1.57-2.57, P=0.000$ ). Simultaneously, the combined HRs of elevated SNHG7 expression on OS in patients with multivariate, K-M Curve respectively were $2.06(95 \%=1.37-3.10, P=0.001)$ and $1.95(95 \%$ $\mathrm{CI}=1.58-2.41, P=0.000)$. Furthermore, in terms of follow-up time, sample sizes and cut off similar results, we observed that cancer patients with worse OS carried high expression of SNHG7 (Figure 3). The above results supported that SNHG7 could be considered as a prognostic indicator, for interfering with OS in cancer patients.

However, only two studies provided data for PFS or DFS, which made it impossible for us to perform a meta-analysis to pool the results. In the study of $\mathrm{Li}$ et al. [23], the HR was 4.505, 95\% CI was 1.98-10.309 $(P=0.0043)$. In the study of Shen et al. [17], HR was $1.73,95 \%$ CI was $1.03-2.90(P<0.001)$. These two studies demonstrated that high SNHG7 expression was significantly related to poor DFS or shorter PFS.

\section{The SNHG7 expression and clinicopathological characteristics}

A further meta-analysis of studies describing tumor characteristics was performed to determine whether the SNHG7 expression was related to the clinicalpathological parameters, including age, gender, TNM stage, lymph node metastases, distant metastasis, and tumor differentiation. The significant association between SNGH7 and lymph node metastases was proved by ten studies ten including 685 patients (positive vs. negative, $\mathrm{OR}=3.32,95 \% \mathrm{CI}=1.61-6.83$, $P=0.001$, Table 3 and Figure 4A). To solve the high heterogeneity, we chose the random-effects model $\left(I^{2}=75 \%, P=0.000\right)$. Meanwhile, six studies involving 515 patients were included to evaluate the connection between SNHG7 and the TNM stage. There was no significantly heterogeneity difference in the studies $\left(I^{2}=18.5 \%, P=0.293\right)$. The pooled $\mathrm{OR}$ (the value was $3.31,95 \%$ CI: $2.29-4.80, P=0.000$ ) indicated that high expression level of SNHG7 was associated with advanced clinical stage. (Figure 4B).

There were five studies describing how SNGH7 involved in histological grading. The analysis showed that OR was $1.92,95 \% \mathrm{CI}$ was $1.22-3.03(P=0.005$, Figure 4C). These data supported poor histological grade was accompanied with elevated SNGH7 expression. A fixed-effects model was used for no heterogeneity in this analysis $\left(I^{2}=13.9 \% ; P=0.325\right)$. There was no statistical significance observed in $\mathrm{DM}(\mathrm{OR}=1.54,95 \% \mathrm{CI}=0.41-5.72, P=0.522)$, age $(\geq 60$ vs. $<60, \mathrm{OR}=1.10,95 \%$ CI: $0.77-1.55$, $P=0.604$ ), and gender (female vs. male, $\mathrm{OR}=1.26$, 95\% CI: 0.95-1.69, $P=0.113$ ) (Figure 4D-4F).

\section{Sensitivity analysis and publication bias}

Sensitivity analysis was to examine whether the OS was interfered by individual studies. There was no significant influence on the pooled results when any 

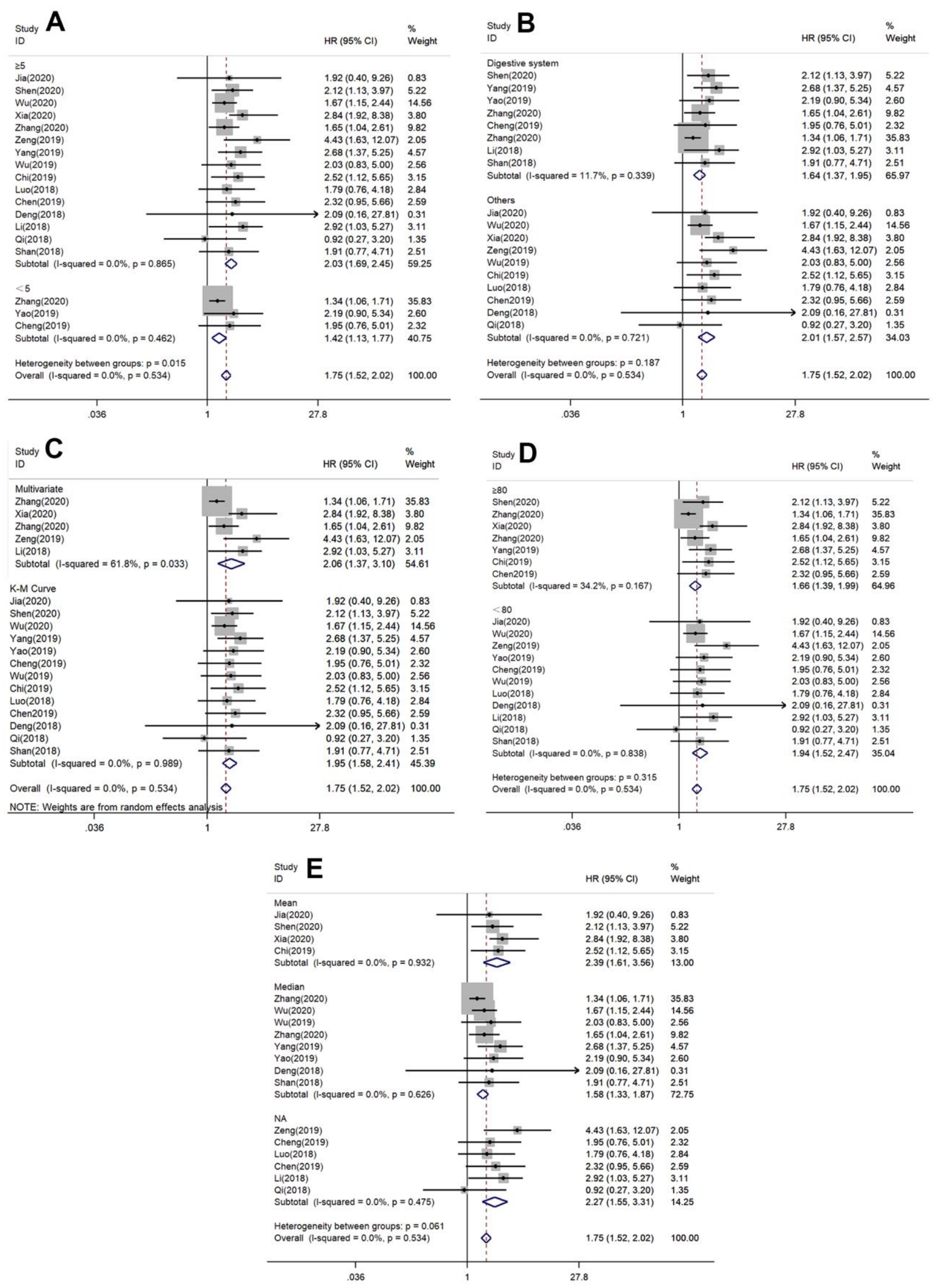

Figure 3. Forest plots of hazard ratios (HRs) for the relationship between SNHG7 expression and overall survival (OS): (A) stratified by followup time; (B) stratified by cancer type; (C) stratified by analysis type; (D) stratified by sample size; (E) stratified by cut off value. 
Table 3. The correlation between SNHG7 expression and clinicopathological features.

\begin{tabular}{|c|c|c|c|c|c|c|c|}
\hline \multirow{2}{*}{ Clinicopathological features } & \multirow{2}{*}{$\begin{array}{l}\text { No. of } \\
\text { studies }\end{array}$} & \multirow{2}{*}{$\begin{array}{c}\text { No. of } \\
\text { patients }\end{array}$} & \multirow{2}{*}{ OR $(95 \% \mathrm{CI})$} & \multirow{2}{*}{$\boldsymbol{P}$} & \multicolumn{2}{|c|}{ Heterogeneity } & \multirow{2}{*}{ Model } \\
\hline & & & & & $I^{2}$ & $P$-value & \\
\hline Age $(\geq 60$ vs. $<60)$ & 7 & 555 & $1.10(0.77-1.55)$ & 0.604 & $0.0 \%$ & 0.714 & Fixed \\
\hline $\begin{array}{l}\text { Gender } \\
\text { (Female vs. male) }\end{array}$ & 11 & 873 & $1.26(0.95-1.69)$ & 0.113 & $0.0 \%$ & 0.800 & Fixed \\
\hline $\begin{array}{l}\text { TNM stage } \\
\text { (III-IV vs. I-II) }\end{array}$ & 6 & 515 & $3.31(2.29-4.80)$ & 0.000 & $18.5 \%$ & 0.293 & Fixed \\
\hline $\begin{array}{l}\text { Lymph node metastases } \\
\text { (Positive vs. negative) }\end{array}$ & 10 & 685 & $3.32(1.61-6.83)$ & 0.001 & $75 \%$ & 0.000 & Random \\
\hline $\begin{array}{l}\text { Distant metastasis } \\
\text { (Positive vs. negative) }\end{array}$ & 6 & 478 & $1.54(0.41-5.72)$ & 0.522 & $84.8 \%$ & 0.000 & Random \\
\hline $\begin{array}{l}\text { Tumor differentiation } \\
\text { (Poor vs. Moderate/Well) }\end{array}$ & 5 & 320 & $1.92(1.22-3.03)$ & 0.005 & $13.9 \%$ & 0.325 & Fixed \\
\hline
\end{tabular}
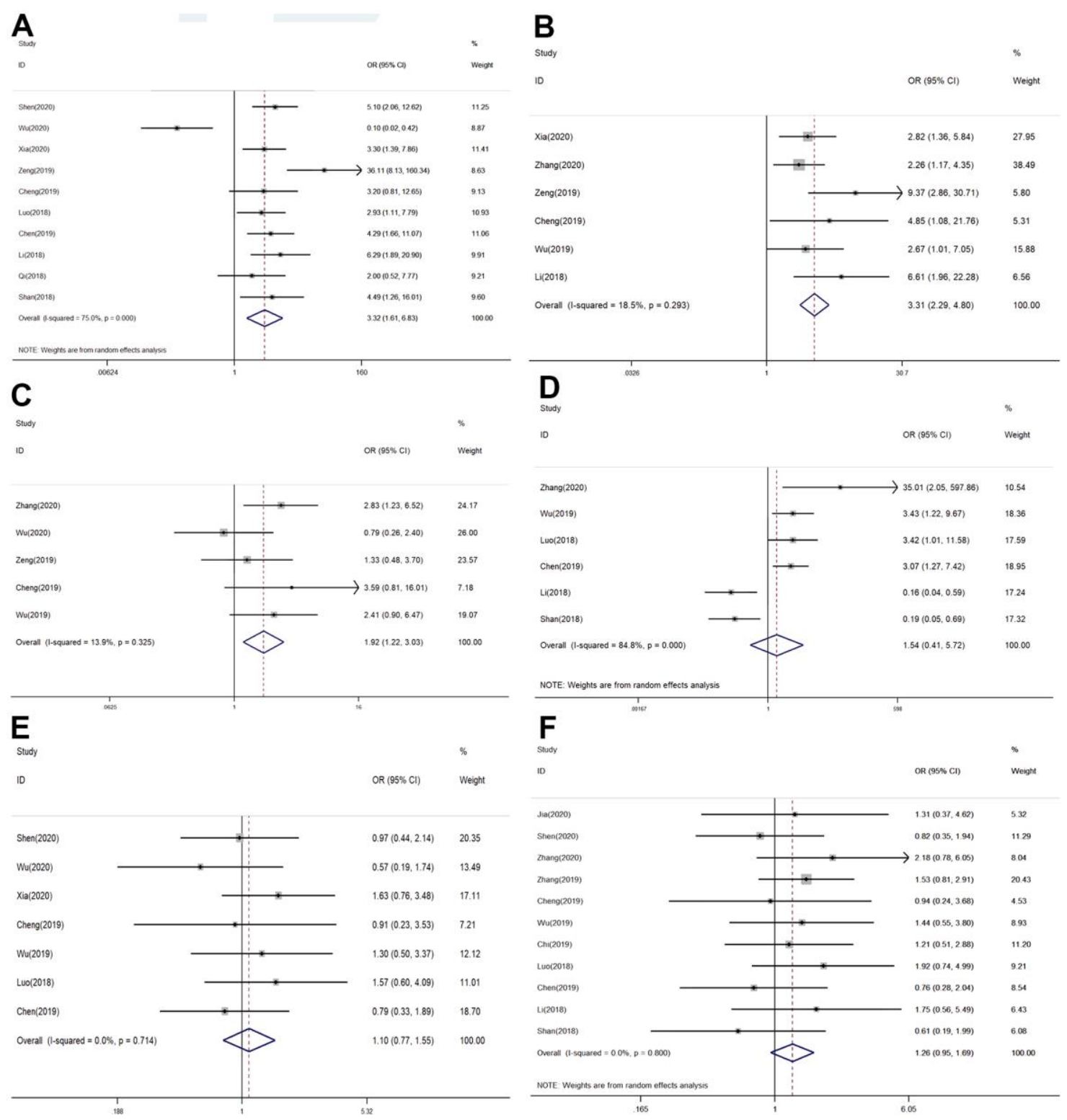

Figure 4. Forest plots of odds ratios (ORs) for the relationship between SNHG7 expression and clinical parameters: (A) lymph node metastasis; (B) TNM stage; (C) Tumor differentiation; (D) distant metastasis; (E) age; (F) gender. 
individual study was removed. At last, we found that the HR values and the synthesized results were stable and reliable (Figure 5A). According to Begg's tests, the symmetrical shape of the funnel plot indicated that no significant publication bias existed in the study $(P=0.649$, Figure 5B).

\section{Validation of TCGA dataset results}

Using the expression data of SNHG7 from the TCGA dataset was to further validate its prognostic and clinical value in cancers. As shown in Figure 6, SNHG7 overexpression was identified in cholangiocarcinoma

A

Meta-analysis fixed-effects estimates (exponential form) Study ommited

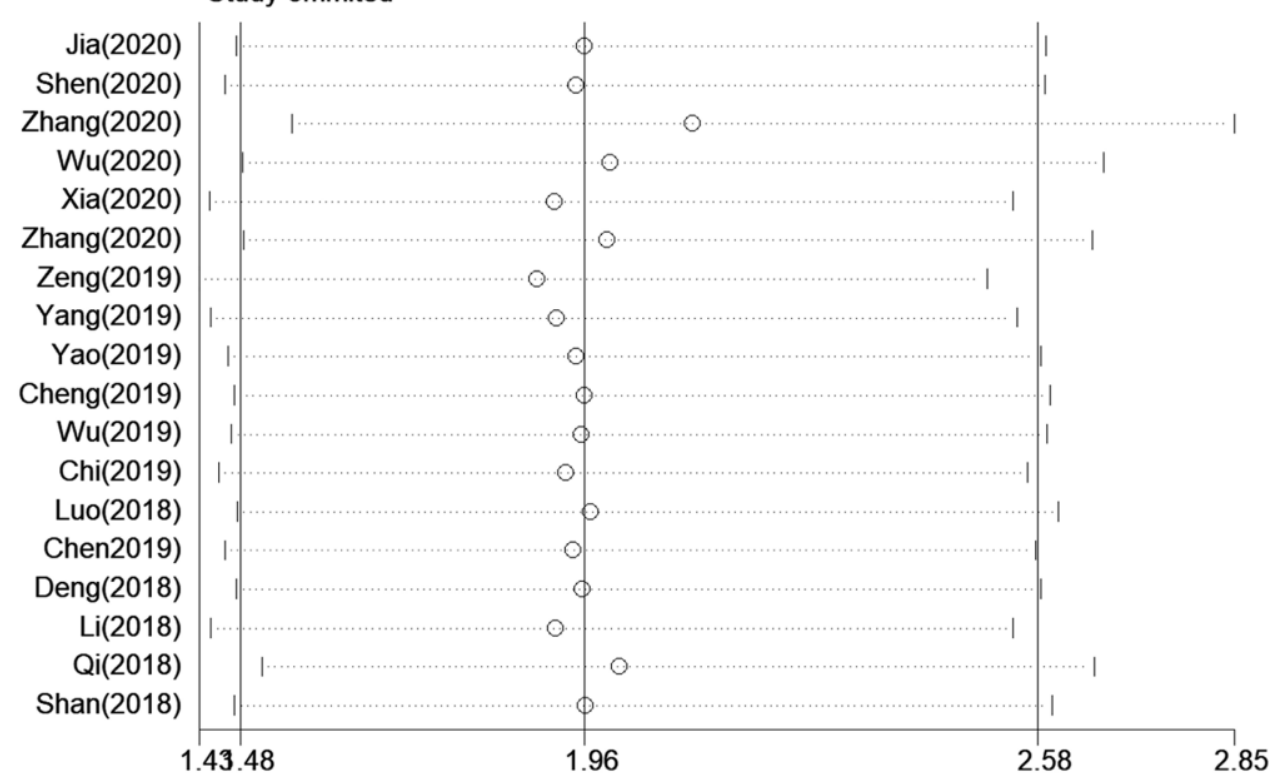

B

Begg's funnel plot with pseudo $95 \%$ confidence limits

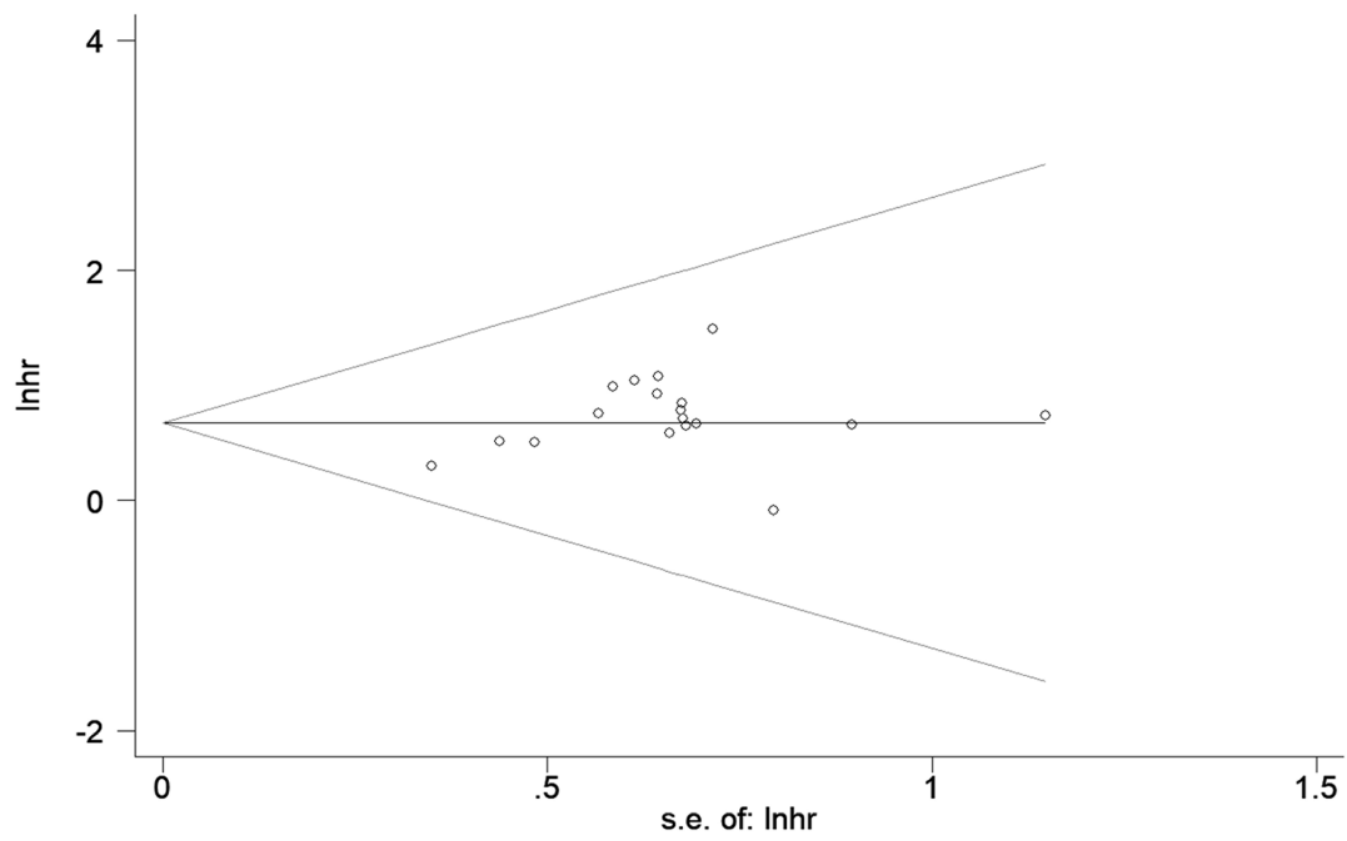

Figure 5. Sensitivity analysis and publication bias for meta-analysis of SNHG7 and OS. (A) Sensitivity analysis for meta-analysis of SNHG7 and OS. (B) Funnel plot of the publication bias for OS. 
(CHOL), colon adenocarcinoma (COAD), liver hepatocellular carcinoma (LIHC), pheochromocytoma and paraganglioma (PCPG) (|Log2fold change (FC)| cutoff $>1$ and $P<0.01)$. Moreover, the violin plot showed that SNHG7 expression was also significantly associated with the clinical stage of human cancers $(P<0.05$, Figure 7). For clarifying the relationship between SNHG7 expression and prognosis, we used the

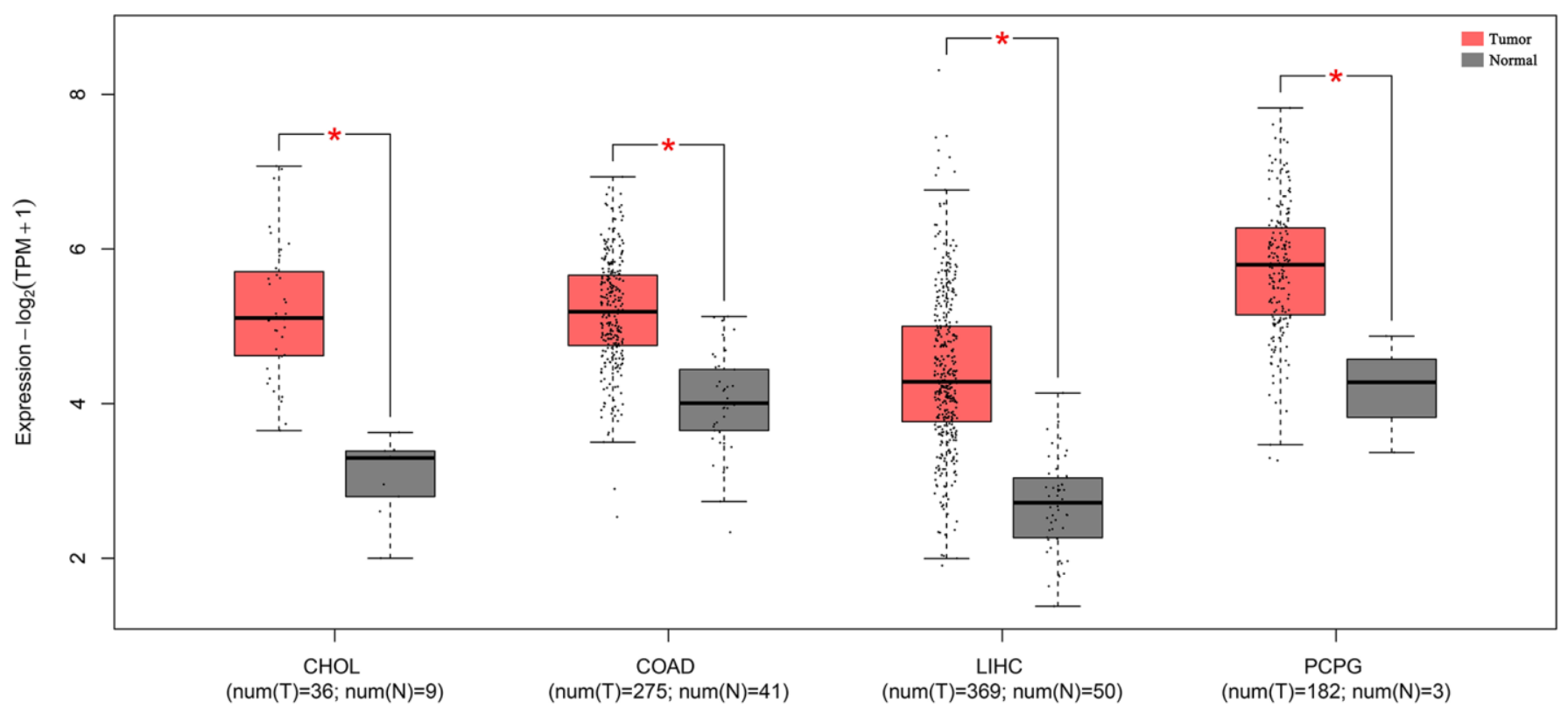

Figure 6. The expression levels of SNHG7 in four kinds of cancer tissues and normal tissues. "*” $|\mathrm{Log} 2 \mathrm{FC}|>1$ and $\mathrm{P}<0.01$. Abbreviations: $\mathrm{CHOL}$, cholangiocarcinoma; COAD, colon adenocarcinoma; LIHC, liver hepatocellular carcinoma; PCPG, pheochromocytoma and paraganglioma.

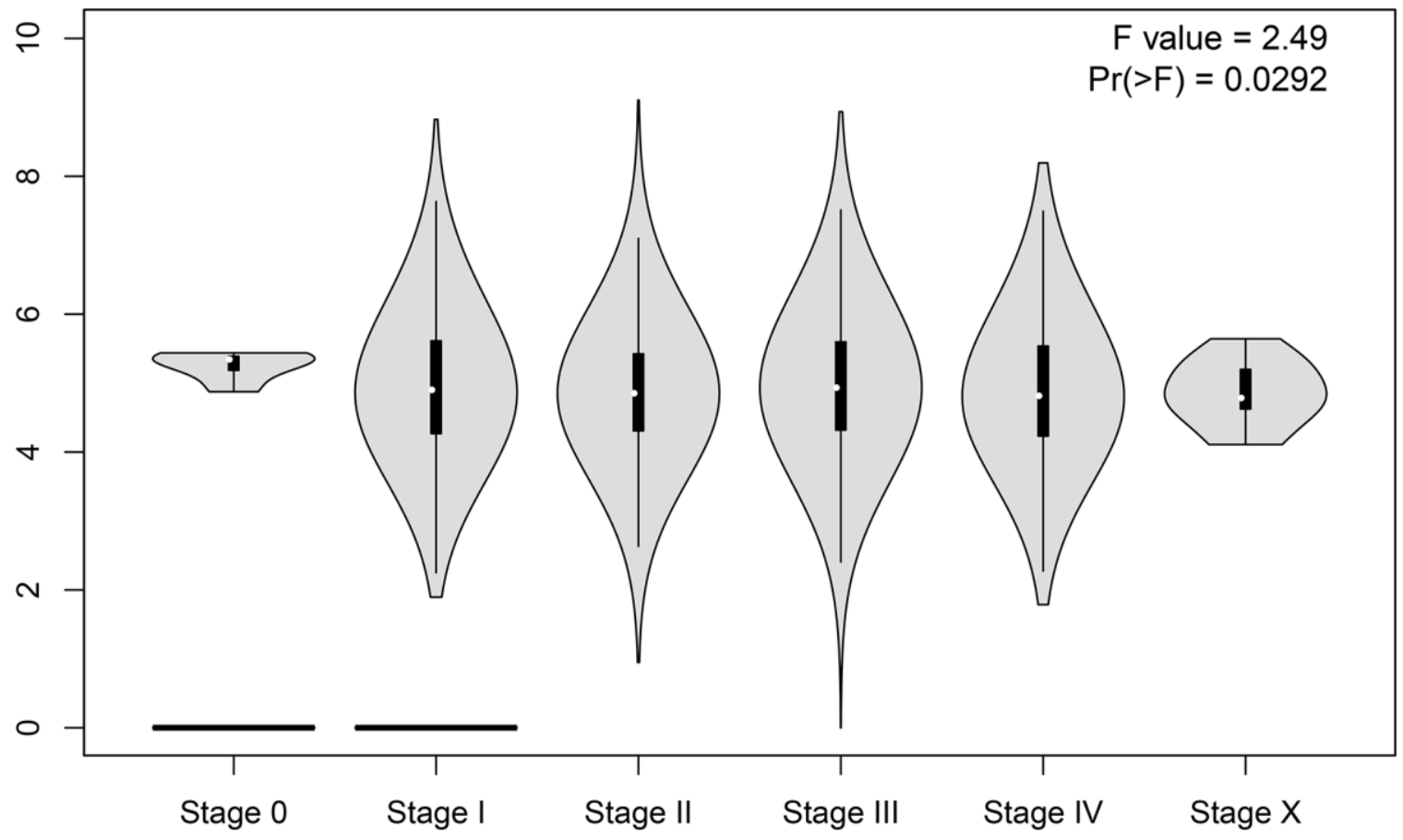

Figure 7. Violin plot of clinical stage of SNHG7 expression in human pancancers. 
GEPIA database to plot survival curve according to quartile grouping cut-off. It has revealed that SNHG7 upregulation was correlated with worse OS in COAD, bladder urothelial carcinoma (BLCA), LIHC (log-rank $P<0.05$, Figure 8 ), which partially confirmed our results in this meta-analysis.

\section{DISCUSSION}

LncRNAs, a biomolecule with regard to tumor epigenetics, post transcriptional regulation, genome stability and ceRNA regulation, has increasingly been emphasized by oncology researchers [37, 38]. LncRNA can act as an oncogene or tumor suppressor gene, and directly or indirectly regulate cancer-related signal pathways to affect tumor development. For example, Liu et al. proved that IncRNA GASL1 inhibited cell proliferation and metastasis by sponging microRNA106a in gastric carcinoma [39]. Xiang et al. confirmed that SNHG7, as an endogenous ceRNA, could regulate HK2 expression via sponging miR-143-3p to promote the proliferation of bladder cancer [40]. Besides, in hepatocellular carcinoma, IncRNA NKILA has been reported for suppressing NF- $\kappa \mathrm{B} /$ Slug pathway mediated with epithelial-mesenchymal transition to inhibit tumor metastasis [41].

SNHG7 was considered as a novel oncogenic lncRNA for its abnormal expression in various types of cancer. A growing number of articles have shown how SNHG7 participated in running processes of molecular biological pathways in the occurrence and development of cancer. Li et al. [23] discovered that the expression of SNHG7 was dramatically up-regulated in hepatocellular carcinoma (HCC), while knockdown of SNHG7 expression could suppress the growth and metastasis of
HCC cells [30]. In breast cancer (BC), some researchers suggested that the increased expression of SNHG7 could promote tumorigenesis and progression by EMT initiation and the activation of the Notch-1 pathway through interacting with miR-34a [42]. In addition, it has been reported that SNHG7 may conduct as an oncogene in colorectal cancer (CRC) progression, and increased expression of SNHG7 is related to cell proliferation, inhibition of cell apoptosis, and enhancement of liver metastasis of CRC [36]. It has proved that SNHG7 was able to regulate osteosarcoma (OS) cell vitality, migration, and invasion by activating sponging miR-34a, which targeted and suppressed the epithelial-mesenchymal transition (EMT) through the TGF- $\beta /$ SMAD4 signaling pathway [35]. Through downregulating expression of SNHG7, Wang et al. found that the proliferation of thyroid cancer (TC) cells could be inhibited and cell apoptosis was induced. Moreover, they indicated that the high expression of SNHG7 was positively correlated with increased tumor size and advanced TNM stage [43]. The study by Chen et al. revealed that the knockdown of SNHG7 expression promoted bladder cancer (BC) cell survival and proliferation via activating the ERK/MAPK signaling pathway [33]. In summary, we made conclusion that SNHG7 plays inconsistent roles in different cancer. Thus, a comprehensive analysis was performed to evaluate whether SNHG7 has the value of predicting the prognosis of tumor patients.

In our finding, lncRNA SNHG7 might be considered as an unsatisfactory prognosis factor for cancer patients. It is inferred that higher expression of SNHG7 would lead to poorer OS, the HR and 95\% CI were 1.75 and 1.52 2.02 respectively. The subgroup meta-analysis stratified by follow-up time, cancer types, analysis types, sample
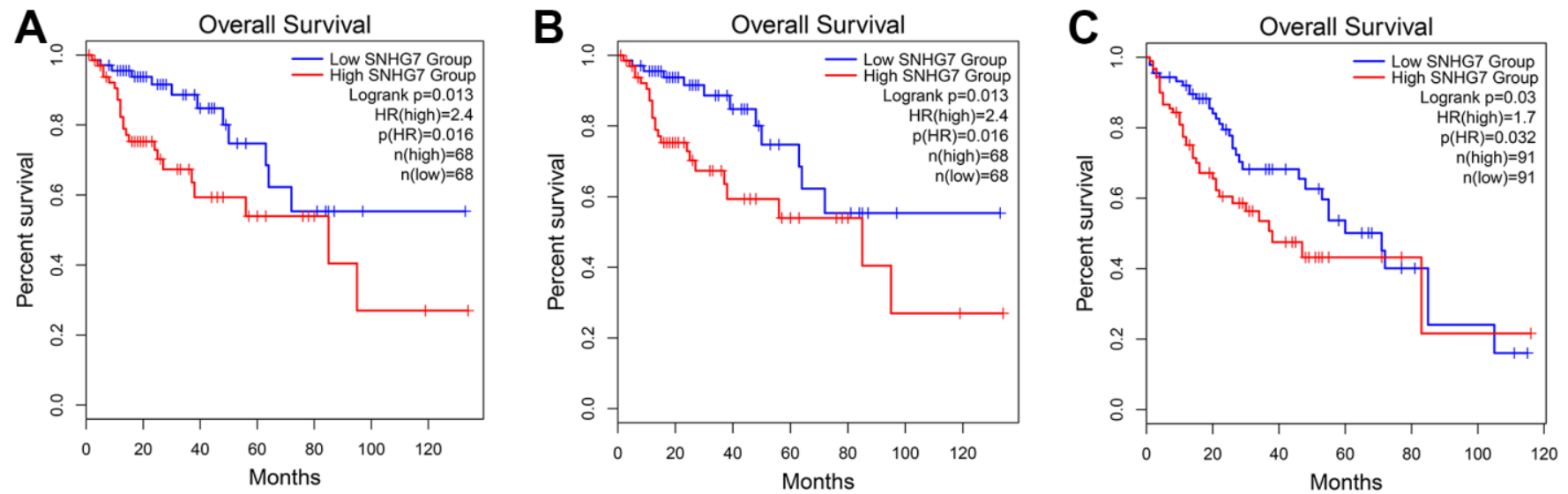

Figure 8. Validation of the prognostic value of SNHG7 based on the TCGA database. (A) The survival curve of patients with COAD; (B) The survival curve of patients with BLCA; (C) the survival curve of patients with LIHC. Abbreviations: COAD, colon adenocarcinoma; BLCA, bladder urothelial carcinoma; LIHC, liver hepatocellular carcinoma; TCGA, The Cancer Genome Atlas. 
sizes and cut off, also drew the above similar conclusions. Notably, the results from TCGA databases also indicated that in colorectal cancer, bladder cancer and hepatocellular carcinoma, the OS of patients with high SNHG7 expression was shorter than that of those with low SNHG7 expression, which partially identified and strengthened our results in this meta-analysis. Moreover, we explored the relationship between SNHG7 expression and clinical parameters. It was noticed that the exiting data supported a significant positive correlation between higher SNHG7 expression level and more advanced TNM, earlier lymph node metastasis, and poorer histological grade. Collectively, we believed that IncRNA SNHG7 was involved in the tumor development and progression, and may become a promising biomarker for prognosis in cancer patients.

Nevertheless, this study has some limitations that should be refined. First of all, due to the relatively small number of included patients, the study might be the lack of certain stringency. Second, the cut-off values including median, mean, and others for positive SNHG7 expression were not consistent in different studies, and lacking a uniform criterion. Third, all patients included in the studies were of Chinese descent. Fourth, the accuracy of HRs and 95\% CIs extracted from KaplanMeier curves were not enough, it might be less than the data directly given in original articles. It might add the potential bias. Hence, the results should be interpreted cautiously.

\section{CONCLUSIONS}

In summary, the higher lncRNA SNHG7 expression would lead to more advanced TNM, earlier lymph node metastasis, and poor histological grade. Thus, cancer patients with poorer OS were often accompanied with the increasing expression of lncRNA SNHG7. Our meta-analysis suggested that lncRNA SNHG7 could serve as a promising biomarker for prognosis with cancers. Still, all in all, we hope that some multi-centre, larger and higher-quality studies will be carried out to strengthen our results in the future.

\section{AUTHOR CONTRIBUTIONS}

Director B. Z. and S. D. designed the study and edited the manuscript. J. W. contributed to drafting the manuscript. C. W. and Z. Z. collected and analyzed the data. All authors have read and approved the final submitted manuscript.

\section{CONFLICTS OF INTEREST}

The authors declare that they have no conflicts of interest.

\section{FUNDING}

This study was supported by National Natural Science Foundation of China (82000842); Guangdong Basic and Applied Basic Research Foundation (2021A1515010151); Project of Administration of Traditional Chinese Medicine of Guangdong Province of China (20211403); Social Science and Technology Development Project of Dongguan (202050715001197).

\section{Editorial note}

\&This corresponding author has a verified history of publications using a personal email address for correspondence.

\section{REFERENCES}

1. Bray F, Ferlay J, Soerjomataram I, Siegel RL, Torre LA, Jemal A. Global cancer statistics 2018: GLOBOCAN estimates of incidence and mortality worldwide for 36 cancers in 185 countries. CA Cancer J Clin. 2018; 68:394-424.

https://doi.org/10.3322/caac.21492 PMID:30207593

2. Miller $K D$, Nogueira $L$, Mariotto $A B$, Rowland $J H$, Yabroff KR, Alfano CM, Jemal A, Kramer JL, Siegel RL. Cancer treatment and survivorship statistics, 2019. CA Cancer J Clin. 2019; 69:363-85. https://doi.org/10.3322/caac.21565 PMID:31184787

3. Soleimani MA, Bahrami N, Allen KA, Alimoradi Z. Death anxiety in patients with cancer: A systematic review and meta-analysis. Eur J Oncol Nurs. 2020; 48:101803. https://doi.org/10.1016/j.ejon.2020.101803 PMID:32836000

4. Torre LA, Siegel RL, Ward EM, Jemal A. Global Cancer Incidence and Mortality Rates and Trends--An Update. Cancer Epidemiol Biomarkers Prev. 2016; 25:16-27. https://doi.org/10.1158/1055-9965.EPI-15-0578 PMID:26667886

5. Quinn JJ, Chang HY. Unique features of long noncoding RNA biogenesis and function. Nat Rev Genet. 2016; 17:47-62.

https://doi.org/10.1038/nrg.2015.10 PMID:26666209

6. Weidle UH, Birzele F, Kollmorgen G, Rüger R. Long Non-coding RNAs and their Role in Metastasis. Cancer Genomics Proteomics. 2017; 14:143-60.

https://doi.org/10.21873/cgp.20027 PMID:28446530

7. Xu T, Lin CM, Cheng SQ, Min J, Li L, Meng XM, Huang C, Zhang L, Deng ZY, Li J. Pathological bases and clinical impact of long noncoding RNAs in prostate cancer: a new budding star. Mol Cancer. 2018; 17:103. 
https://doi.org/10.1186/s12943-018-0852-7 PMID:30037351

8. Fatica A, Bozzoni I. Long non-coding RNAs: new players in cell differentiation and development. Nat Rev Genet. 2014; 15:7-21. https://doi.org/10.1038/nrg3606 PMID:24296535

9. Schmitt AM, Chang HY. Long Noncoding RNAs in Cancer Pathways. Cancer Cell. 2016; 29:452-63. https://doi.org/10.1016/j.ccell.2016.03.010 PMID:27070700

10. Shengnan J, Dafei X, Hua J, Sunfu F, Xiaowei W, Liang X. Long non-coding RNA HOTAIR as a competitive endogenous RNA to sponge miR-206 to promote colorectal cancer progression by activating CCL2. J Cancer. 2020; 11:4431-41.

https://doi.org/10.7150/ica.42308

PMID:32489462

11. Zhang JF, Jiang $\mathrm{W}$, Zhang QF, Kuai XL, Mao ZB, Wang ZW. Long noncoding RNA STCAT16 suppresses cell growth and its expression predicts prognosis in patients with gastric cancer. Mol Med Rep. 2019; 19:4613-22.

https://doi.org/10.3892/mmr.2019.10128

PMID: $\underline{30957180}$

12. Huang HW, Xie H, Ma X, Zhao F, Gao Y. Upregulation of LnCRNA PANDAR predicts poor prognosis and promotes cell proliferation in cervical cancer. Eur Rev Med Pharmacol Sci. 2017; 21:4529-35.

PMID:29131264

13. Pardini B, Sabo AA, Birolo G, Calin GA. Noncoding RNAs in Extracellular Fluids as Cancer Biomarkers: The New Frontier of Liquid Biopsies. Cancers (Basel). 2019; 11:1170.

https://doi.org/10.3390/cancers11081170

PMID:31416190

14. Dragomir MP, Kopetz S, Ajani JA, Calin GA. Non-coding RNAs in GI cancers: from cancer hallmarks to clinical utility. Gut. 2020; 69:748-63.

https://doi.org/10.1136/gutinl-2019-318279

PMID:32034004

15. Luo $X$, Song $Y$, Tang L, Sun DH, Ji DG. LncRNA SNHG7 promotes development of breast cancer by regulating microRNA-186. Eur Rev Med Pharmacol Sci. 2018; 22:7788-97.

https://doi.org/10.26355/eurrev 201811_16403 PMID:30536320

16. Cheng D, Fan J, Ma Y, Zhou Y, Qin K, Shi M, Yang J. LncRNA SNHG7 promotes pancreatic cancer proliferation through ID4 by sponging miR-342-3p. Cell Biosci. 2019; 9:28. https://doi.org/10.1186/s13578-019-0290-2
PMID:30949340

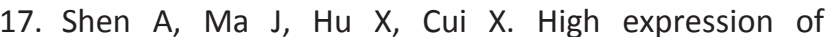
IncRNA-SNHG7 is associated with poor prognosis in hepatocellular carcinoma. Oncol Lett. 2020; 19:3959-63.

https://doi.org/10.3892/ol.2020.11490

PMID:32382340

18. Wu F, Sui Y, Wang Y, Xu T, Fan L, Zhu H. Long Noncoding RNA SNHG7, a Molecular Sponge for microRNA-485, Promotes the Aggressive Behavior of Cervical Cancer by Regulating PAK4. Onco Targets Ther. 2020; 13:685-99. https://doi.org/10.2147/OTT.S232542 PMID:32158221. Retraction in: Onco Targets Ther. 2021; 14:4741-42. https://doi.org/10.2147/OTT.S337553

19. Xu C, Zhou J, Wang Y, Wang A, Su L, Liu S, Kang X. Inhibition of malignant human bladder cancer phenotypes through the down-regulation of the long non-coding RNA SNHG7. J Cancer. 2019; 10:539-46. https://doi.org/10.7150/jca.25507 PMID:30719150

20. She K, Huang J, Zhou H, Huang T, Chen G, He J. IncRNASNHG7 promotes the proliferation, migration and invasion and inhibits apoptosis of lung cancer cells by enhancing the FAIM2 expression. Oncol Rep. 2016; 36:2673-80. https://doi.org/10.3892/or.2016.5105 PMID:27666964

21. Wu X, Yuan Y, Ma R, Xu B, Zhang R. IncRNA SNHG7 affects malignant tumor behaviors through downregulation of EZH2 in uveal melanoma cell lines. Oncol Lett. 2020; 19:1505-15.

https://doi.org/10.3892/ol.2019.11240

PMID:32002036

22. Chi R, Chen X, Liu M, Zhang H, Li F, Fan X, Wang W, Lu $H$. Role of SNHG7-miR-653-5p-STAT2 feedback loop in regulating neuroblastoma progression. J Cell Physiol. 2019; 234:13403-12.

https://doi.org/10.1002/jcp.28017

PMID:30623419

23. Li Y, Zeng C, Hu J, Pan Y, Shan Y, Liu B, Jia L. Long noncoding RNA-SNHG7 acts as a target of miR-34a to increase GALNT7 level and regulate PI3K/Akt/mTOR pathway in colorectal cancer progression. J Hematol Oncol. 2018; 11:89.

https://doi.org/10.1186/s13045-018-0632-2 PMID:29970122

24. Stang A. Critical evaluation of the Newcastle-Ottawa scale for the assessment of the quality of nonrandomized studies in meta-analyses. Eur J Epidemiol. 2010; 25:603-05.

https://doi.org/10.1007/s10654-010-9491-z PMID:20652370

25. Jia J, Zhang D, Zhang J, Yang L, Zhao G, Yang H, Wang J. 
Long non-coding RNA SNHG7 promotes neuroblastoma progression through sponging miR-323a-5p and miR342-5p. Biomed Pharmacother. 2020; 128:110293.

https://doi.org/10.1016/i.biopha.2020.110293

PMID:32534305

26. Zhang P, Shi L, Song L, Long Y, Yuan K, Ding W, Deng L. LncRNA CRNDE and IncRNA SNHG7 are Promising Biomarkers for Prognosis in Synchronous Colorectal Liver Metastasis Following Hepatectomy. Cancer Manag Res. 2020; 12:1681-92.

https://doi.org/10.2147/CMAR.S233147 PMID:32210611

27. Xia Q, Li J, Yang Z, Zhang D, Tian J, Gu B. Long noncoding RNA small nucleolar RNA host gene 7 expression level in prostate cancer tissues predicts the prognosis of patients with prostate cancer. Medicine (Baltimore). 2020; 99:e18993.

https://doi.org/10.1097/MD.0000000000018993 PMID:32049793

28. Zhang Y, Yuan Y, Zhang Y, Cheng L, Zhou X, Chen K. SNHG7 accelerates cell migration and invasion through regulating miR-34a-Snail-EMT axis in gastric cancer. Cell Cycle. 2020; 19:142-52.

https://doi.org/10.1080/15384101.2019.1699753

PMID:31814518

29. Zeng J, Ma YX, Liu ZH, Zeng YL. LncRNA SNHG7 contributes to cell proliferation, invasion and prognosis of cervical cancer. Eur Rev Med Pharmacol Sci. 2019; 23:9277-85.

https://doi.org/10.26355/eurrev 201911 19420 PMID:31773679

30. Yang $X$, Sun L, Wang L, Yao B, Mo H, Yang W. LncRNA SNHG7 accelerates the proliferation, migration and invasion of hepatocellular carcinoma cells via regulating miR-122-5p and RPL4. Biomed Pharmacother. 2019; 118:109386.

https://doi.org/10.1016/i.biopha.2019.109386

PMID:31545291

31. Yao X, Liu C, Liu C, Xi W, Sun S, Gao Z. IncRNA SNHG7 sponges miR-425 to promote proliferation, migration, and invasion of hepatic carcinoma cells via $W n t / \beta-$ catenin/EMT signalling pathway. Cell Biochem Funct. 2019; 37:525-33.

https://doi.org/10.1002/cbf.3429 PMID:31478234

32. Wu P, Tang Y, Fang X, Xie C, Zeng J, Wang W, Zhao S. Metformin Suppresses Hypopharyngeal Cancer Growth by Epigenetically Silencing Long Non-coding RNA SNHG7 in FaDu Cells. Front Pharmacol. 2019; 10:143. https://doi.org/10.3389/fphar.2019.00143 PMID:30853913

33. Chen $Y$, Peng $Y, X u Z$, Ge B, Xiang $X$, Zhang $T$, Gao L, Shi $\mathrm{H}$, Wang $\mathrm{C}$, Huang J. Knockdown of IncRNA SNHG7 inhibited cell proliferation and migration in bladder cancer through activating Wnt/ $\beta$-catenin pathway. Pathol Res Pract. 2019; 215:302-07.

https://doi.org/10.1016/i.prp.2018.11.015

PMID:30527358

34. Qi H, Wen B, Wu Q, Cheng W, Lou J, Wei J, Huang J, Yao X, Weng G. Long noncoding RNA SNHG7 accelerates prostate cancer proliferation and cycle progression through cyclin D1 by sponging miR-503. Biomed Pharmacother. 2018; 102:326-32. https://doi.org/10.1016/j.biopha.2018.03.011 PMID:29571017

35. Deng $Y$, Zhao F, Zhang Z, Sun F, Wang M. Long Noncoding RNA SNHG7 Promotes the Tumor Growth and Epithelial-to-Mesenchymal Transition via Regulation of miR-34a Signals in Osteosarcoma. Cancer Biother Radiopharm. 2018; 33:365-72. https://doi.org/10.1089/cbr.2018.2503 PMID:29989838

36. Shan Y, Ma J, Pan Y, Hu J, Liu B, Jia L. LncRNA SNHG7 sponges miR-216b to promote proliferation and liver metastasis of colorectal cancer through upregulating GALNT1. Cell Death Dis. 2018; 9:722.

https://doi.org/10.1038/s41419-018-0759-7 PMID:29915311

37. Chandra Gupta S, Nandan Tripathi Y. Potential of long non-coding RNAs in cancer patients: From biomarkers to therapeutic targets. Int J Cancer. 2017; 140:1955-67.

https://doi.org/10.1002/ijc.30546

PMID:27925173

38. Huarte $M$. The emerging role of IncRNAs in cancer. Nat Med. 2015; 21:1253-61.

https://doi.org/10.1038/nm.3981

PMID:26540387

39. Liu D, Xiao P, Feng C, Meng H, Bi E. Long non-coding RNA GASL1 restrains gastric carcinoma cell proliferation and metastasis by sponging microRNA106a. Cell Cycle. 2020; 19:2611-21. https://doi.org/10.1080/15384101.2020.1812918 PMID: 32897806

40. Xiang W, Lyu L, Huang T, Zheng F, Yuan J, Zhang C, Jiang $G$. The long non-coding RNA SNHG1 promotes bladder cancer progression by interacting with miR143-3p and EZH2. J Cell Mol Med. 2020; 24:11858-73. https://doi.org/10.1111/jcmm.15806 PMID:32885590

41. Chen R, Cheng Q, Owusu-Ansah KG, Song G, Jiang D, Zhou L, Xu X, Wu J, Zheng S. NKILA, a prognostic indicator, inhibits tumor metastasis by suppressing NF-kB/Slug mediated epithelial-mesenchymal transition in hepatocellular carcinoma. Int J Biol Sci. 
2020; 16:495-503.

https://doi.org/10.7150/ijbs.39582

PMID:32015685

42. Sun $X$, Huang $T$, Liu $Z$, Sun $M$, Luo S. LncRNA SNHG7 contributes to tumorigenesis and progression in breast cancer by interacting with miR-34a through EMT initiation and the Notch-1 pathway. Eur J Pharmacol. 2019; 856:172407.

https://doi.org/10.1016/i.ejphar.2019.172407 PMID:31132353

43. Wang YH, Huo BL, Li C, Ma G, Cao W. Knockdown of long noncoding RNA SNHG7 inhibits the proliferation and promotes apoptosis of thyroid cancer cells by downregulating BDNF. Eur Rev Med Pharmacol Sci. 2019; 23:4815-21.

https://doi.org/10.26355/eurrev_201906_18067

PMID:31210313 